\title{
La mémoire théâtrale de l'avarice dans The Merchant of Venice
}

\section{Tatiana Burtin}

\section{(2) OpenEdition}

Journals

Édition électronique

URL : http://journals.openedition.org/shakespeare/1926

DOI : 10.4000/shakespeare.1926

ISSN : 2271-6424

Éditeur

Société Française Shakespeare

Édition imprimée

Date de publication : 1 avril 2013

Pagination : 107-126

ISBN : 2-9521475-9-0

\section{Référence électronique}

Tatiana Burtin, «La mémoire théâtrale de l'avarice dans The Merchant of Venice », Actes des congrès de

la Société française Shakespeare [En ligne], 30 | 2013, mis en ligne le 03 avril 2013, consulté le 19 avril 2019. URL : http://journals.openedition.org/shakespeare/1926 ; DOI : 10.4000/shakespeare.1926 


\section{Shakespeare}

\section{et la mémoire}

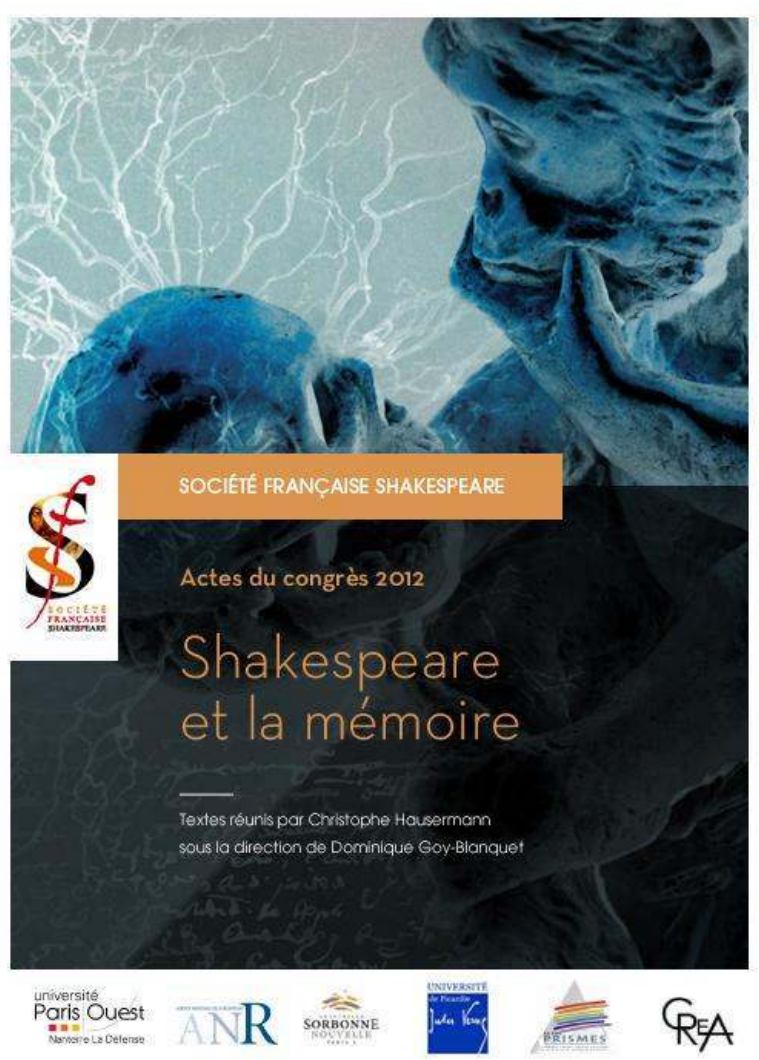

actes du Congrès

organisé par la

SOCIÉTÉ FRANÇAISE SHAKESPEARE

les 22, 23 et 24 mars 2012 
textes réunis par

Christophe HAUSERMANN

sous la direction de

Dominique Goy-Blanguet 


\section{COUVERTURE}

d'après l'affiche de Claire Colombet

conception graphique et logo

Pierre Kapitaniak

mise en page et corrections

Christophe Hausermann 
Institut du Monde Anglophone

Université de Paris III - Sorbonne Nouvelle

5 rue de l’École de Médecine

75006 Paris

www.societefrancaiseshakespeare.org

Tous droits de traduction, de reproduction et d'adaptation

réservés pour tous les pays 


\title{
LA MEMOIRE THEATRALE DE L'AVARICE DANS THE MERCHANT OF VENICE
}

\author{
Tatiana BURTIN
}

\begin{abstract}
Le thème de l'avarice appartient à une tradition de représentation religieuse et théâtrale, qui va des Écritures aux Moralités, en passant par la comédie gréco-latine, jusqu'à son apogée sur la scène classique. L'usurier juif Shylock est une expression très aboutie de cette mémoire représentative de l'avarice. L'intrigue, en relayant l'inquiétude de l'époque quant à la stabilité des valeurs économiques, politiques et culturelles, suspend ce personnage entre le comique et le tragique. Shylock confronte traits comiques antiquisants, allégorie religieuse du théâtre médiéval et topoi ethniques à une révision de la conception de l'usure et de l'intérêt, autrefois dénigrés par la morale chrétienne. Cette construction d'une nouvelle figure de l'avarice déstabilise le genre comique par une revalorisation (ou une dévalorisation?) de ces concepts moraux et économiques et par une hybridation des genres.

The theme of greed belongs to a long tradition of religious and moral literature that goes from the Holy Bible to Morality plays, through Greco-Roman comedy, and peaks in classical drama. Shylock, the Jewish usurer, is perfectly representative of this dramatic memory of avarice. In a plot fraught with contemporary doubts on economic, political and cultural values' stability, Shakespeare's miser and usurer balances between comedy and tragedy. Shylock synthesizes ancient and medieval features of comedy and allegory and ethnical topoi, and brings forth a revised conception of usury and interest, which is set apart from Christian morality. The conventions of comic drama are shaken by this construction of a new figure of avarice: not only a new value is given (or is all value denied?) to these moral and economic concepts, but drama genres are also hybridized. Therefore, a close observation of Shakespeare's art of playwriting is crucial to a better understanding of how he spelled out a puzzling and threatening social context.
\end{abstract}

$\mathrm{B}$

ien que voué à l'éphémère, le spectacle est un art de la mémoire. Mémoire entraînée des comédiens bien sûr, qui s'exerce sur un texte, un jeu, un espace. Mais le théâtre a aussi une mémoire propre, celle de la tradition. Shylock s'inscrit dans une tradition théâtrale de l'avarice et de l'usure qui va de la comédie gréco-romaine au théâtre élisabéthain et qu'il contribue à faire évoluer radicalement. Quelques-uns de ses traits évoquent cette continuité de représentation, mais Shakespeare invente dans The Merchant of Venice ${ }^{1}$ une figure moderne autrement plus complexe, qui, tout en s'appuyant sur des modèles dont l'efficacité comique est éprouvée, fait surgir des questionnements nouveaux sur le rapport de ses contemporains à l'argent et aux valeurs morales et économiques. S'appuyant sur une série de poncifs ethniques ancrés dans la mémoire collective et un

${ }^{1}$ Les références à ce texte sont tirées de l'édition The Norton Shakespeare, fondée sur la Oxford Edition, éd. Stephen Jay Greenblatt et alii, New York, W.W. Norton \& Company, 1997 (désormais abrégée en NS). 
réseau de références littéraires et dramatiques sur le rapport entre judéité et avarice ou, plus largement, malfaisance sociale, remotivées par l'histoire la plus récente, l'œuvre de Shakespeare et le personnage de Shylock sont inscrits dans notre mémoire culturelle comme porteurs d'une vision socio-politique, religieuse et dramatique moderne de l'argent et de ses manipulations.

Nous proposons donc d'analyser comment la mise en scène des rapports à l'argent des personnages du Merchant of Venice, comédie composée entre 1596 et $1597^{2}$, met en crise les concepts éthiques, économiques, politiques et culturels du règne élisabéthain. À travers ces représentations conflictuelles s'esquisse la recherche d'une esthétique comique. Est-il encore possible de rire de l'avare, personnage connu depuis l'Antiquité, allégorisé dans le théâtre médiéval, ethnicisé sous les traits du juif, alors qu'il met plus que jamais en péril les valeurs les plus sacrées de la chrétienté ? Le personnage sort littéralement d'une mémoire littéraire et religieuse sclérosée, pour prendre vie, avec ses contradictions et ses angoisses. Le travail de Shakespeare ne tendrait-il pas dès lors à laïciser la Moralité, à un moment d'épuisement du modèle comique de l'avarice, au cours d'une période de conjonctures religieuses et économiques nouvelles, marquée par les conflits entre protestants et catholiques, le développement d'une économie de marché, le débat sur la condamnation chrétienne de l'usure, la réorganisation du pouvoir et de l'État par les Tudors, mais aussi la professionnalisation des métiers du spectacle? La construction d'une telle figure ouvre dans l'espace de la comédie de l'avarice la fissure angoissante du tragique, et fait réfléchir son auteur sur le genre comique lui-même.

${ }^{2}$ La pièce (ci-après abrégée en $M V$ ) est inscrite sur le Registre des Libraires en juillet 1598 , puis en octobre 1600 , où elle paraît pour la première fois en in-quarto (Q1). Un second inquarto est publié en 1619 (Q2), puis la pièce est reprise en in-folio dans la première édition des Euvres complètes de Shakespeare parue en 1623 (F1). 


\section{Shylock dans la « mémoire » théâtrale de l'avarice}

Le thème de l'avarice a été abondamment traité hors du théâtre, mais sa vulgarité, au sens étymologique du terme, en fait volontiers un sujet de comédie. Pour les philosophes grecs ${ }^{3}$, l'excès dans l'amour des richesses est un vice à la fois politique et moral, et c'est ainsi qu'il se voit porté sur la scène dès la période attique. La Néa semble insister davantage que la comédie d'Aristophane sur la moralité ou l'amoralité de ses personnages ; nous possédons peu d'exemples de cette forme de comédie de mœurs. Le « dyscolos » de Ménandre est considéré depuis longtemps comme un ancêtre des vieillards misanthropes et avares ${ }^{4}$. L'Euclion de Plaute, probablement issu d'une autre comédie de Ménandre 5 , cumule un excès et un défaut propres à sa famille, non à la cité : il est avarus (avide, cupide) par son grand-père, celui qui a trouvé et caché une marmite pleine d'or au cœur de son foyer, au lieu de la dépenser pour le bien de ses enfants ; et il est parcus (pingre) par son père, car celui-ci, faute d'avoir honoré comme il se doit le lare de son foyer, s'est vu privé de la connaissance du trésor qu'il abrite ${ }^{6}$. Son caractère est mis en parallèle avec celui de Mégadore, un bourgeois misogyne pour les besoins de la comédie, qui ne perd pas de vue son profit : il fait l'éloge de la parsimonia dans un long monologue, où il démontre qu'une femme pauvre épargne à son mari des dépenses coûteuses supplémentaires, car le mariage est déjà un fardeau en soi 7 .

Déjà chez Plaute, tout est une question d'économie, c'est-à-dire d'équilibre dans la gestion domestique. Euclion, en retenant "l'héritage » de son aïeul et du lare, perturbe cette juste économie, et c'est ce qui le rend avare, et comique. La révélation du trésor le fait sombrer dans une sorte de folie, de maladie (« intemperiae », I.i.28;

\footnotetext{
3 Platon, Lois, vi, 754e, Paris, Flammarion, 2006, p. 289; Xénophon, Économique, chap. xiv, 4-10, Paris, Les Belles Lettres, 1949, p. 90-91; Aristote, Éthique à Nicomaque, IV.4, Paris, Flammarion, 2008, p. 121-132.

${ }^{4}$ Ménandre, Le Dyscolos, $2^{\text {ème }}$ éd. J-M. Jacques, Paris, Les Belles Lettres, 1976. Voir l'étude de Max Bonnet, "Smikrinès - Euclion - Harpagon », Mélanges Louis Havet - Philologie et Linguistique, Paris, Hachette, 1909, p. 17-37.

5 Eckart Lefèvre, «L'Aulularia de Plaute, L'Avare de Molière et la version originale grecque : des formes différentes du comique », Ktèma 22, 1997, p. 227-235.

${ }^{6}$ Benjamín García Hernandez, " Euclio (Plauto, Aulularia), parcus atque avarus », Emerita 72, vol. 2, 2004, p. 227-248.

7 Plaute, Aulularia, III.v.475-535, in Comédies, tome I, éd. Alfred Ernout [1 ${ }^{\text {re }}$ éd. 1932], «Les Belles Lettres », Paris, Collection Universitaire de France, 1963, p. 176-178.
} 
« mala crux te agitat », IV.x.631 ; « insanis », IV.x.653; « sanis tu non es », IV.x.769) : il ne dort plus, ne mange plus, regarde à toutes les dépenses. Il ne recouvre la santé qu'avec le don de la marmite à sa fille. Pour l'usurier de Shakespeare, c'est le vol de ses ducats et la fuite de sa fille qui perturbe l'économie de sa « sobre demeure " (sober house) ${ }^{8}$. Donner ses ducats, marier sa fille à un chrétien revient à contrer tous les moyens de son action dans Venise, à remplacer l'usure par le don généreux, mais aussi à assimiler de force l'usurier juif à la foi et à l'économie chrétienne et marchande.

On peut objecter que l'avare de l'Aulularia n'a rien à voir avec Shylock 9 . Ainsi Arthur Stonex, dans un article paru en 1916 dans les Publications of the Modern Language Association ${ }^{10}$, considère que la figure du juif usurier dans le théâtre élisabéthain ne dérive pas des rôles similaires de la comédie plautinienne (avare / avidus ou parcus, prêteur sur gages / danista, banquier / trapezita), car ils n'attirent pas la même réprobation sur leurs actes. Le leno (le proxénète) serait peutêtre le plus proche de cette détestation :

After finding that many Elizabethan descriptions of the physical appearance, the dress, and the personal habits of the usurer were modelled closely on medieval descriptions of Avarice, particularly upon realizing the close spiritual affinity between the two, I was led to look to the Avaritia who appears so often in the morality plays as the prototype of the usurer of the later drama. And here it is possible to trace a line of descent, but a line so faint and uncertain that it can be suggested as only a not improbable hypothesis ${ }^{11}$.

${ }^{8} M V$, II.v.35.

${ }^{9}$ Même s'il est possible de repérer une inspiration plautinienne dans certains passages ; voir Tatiana Burtin, Figures de l'avarice et de l'usure dans les comédies : The Merchant of Venice de Shakespeare, Volpone de Ben Jonson et L'Avare de Molière, thèse de doctorat, Université de Montréal et de Paris Ouest Nanterre, 2011, p. 27-28 et Véronique Marcou, L'ambivalence de l'or à la Renaissance. Ronsard, d'Aubigné, Shakespeare, Paris, L'Harmattan, 1998, p. 152-153.

10 Arthur B. Stonex, « The Usurer in Elizabethan Drama », PMLA 31.2, 1916, p. 190-210.

${ }^{11}$ Stonex, op. cit., p. 192-93 (c'est moi qui souligne). À notre connaissance, aucun autre article plus récent n'étudie cette lignée entre les anciennes allégories théâtrales de l'avarice et Shylock. Ce lieu commun est si profondément ancré dans notre mémoire culturelle qu'il ne semble plus faire débat dans la réflexion contemporaine, qui préfère s'attarder sur les représentations des perspectives économiques à travers textes et milieux littéraires. C'est notamment le cas du "New Economic Criticism ». Le terme fait son apparition dans l'ouvrage collectif édité par Martha Woodmansee et Mark Osteen, The New Economic Criticism: Studies at the Intersection of Literature and Economics, New-York, Routledge, 1999. Cette première génération de critiques étudie la reconceptualisation de l'argent 
Il y aurait donc une lignée de la description de l'avarice, des " morality plays » à Shylock. Si Stonex observe tant de prudence, c'est qu'en effet la lignée est loin d'être claire si l'on considère le déroulement de l'intrigue : Respublica (moralité « politique » de 1553) campe une Avarice qui « remplit ses treize bourses des intérêts de ses usures "; Greedinesse dans The Tide Tarrieth No Man de George Wapull ${ }^{12}$ (1576 ?) est clairement un usurier, accompagné de ses associés Corage, « The Vice » et Hurtfull-Helpe ; mais aucune moralité ne connaît le mécanisme qui renversera par la suite l'usurier élisabéthain tel qu'il apparaît dans The Jew of Malta (1589-1590), et dans The Merchant of Venice ${ }^{13}$. L'Usure reste une abstraction à vocation édifiante dans The Three Ladies of London (1583) et The Three Lords and Ladies of London (1585-1588) de Robert Wilson ${ }^{14}$, où elle est pourtant arrêtée après une série de crimes contre PlainDealing, Hospitality, Liberality et Conscience, puis marquée au fer d'un petit « $\mathrm{x}$ » au milieu d'un grand $\mathrm{C}$, pour signifier qu'on ne peut demander un taux d'intérêt de 10\%. L'Usurier de A Looking Glass for London and England de Lodge et Greene ${ }^{15}$ (1589) est déjà moins abstrait: il défait Thrasybule, un jeune gentilhomme, et Alcon, un pauvre paysan, puis doit rendre justice de ses gains illégaux, terrassé par la peur de voir apparaître les fantômes de ses victimes. Avec Barabas et Shylock, l'usurier gagne un nom ${ }^{16}$. Stonex se fonde

(money), du trésor au capital, et la refiguration consécutive de la matière argent (specie) en papier à partir du XVIII ${ }^{\mathrm{e}}$ siècle (voir chapitre "Taking Account of the New Economic Criticism: An Historical Introduction", p. 5), notamment visibles dans les romans (voir, James Thompson, Models of Value: Eighteenth-Century Politic Economy and the Novel, Durham, Duke University Press, 1996). Pour la recherche française, il faut citer l'ouvrage fondamental de Pierre Bourdieu, Les Règles de l'art. Genèse et structure du champ littéraire [1992], Paris, Seuil, 1998, qui se penche sur les conditions de production du roman au XIX ${ }^{\mathrm{e}}$ siècle. L'ouvrage dirigé par Linda Woodbridge, Money and the Age of Shakespeare: Essays in New Economic Criticism, New-York, Palgrave Macmillan, 2003, se distingue des travaux précédents par l'éclairage qu'il porte sur le théâtre élisabéthain.

${ }^{12}$ George Wapull, The Tide Tarrieth No Man, [1576?], Londres, Tudor Facsimile Texts, 1910 .

13 Stonex, op. cit., p. 195-196. Il accorde d'ailleurs étrangement fort peu de place à l'analyse de Shakespeare (p. 198).

14 Robert Wilson, The Three Ladies of London, [1584] et The Three Lords and The Three Ladies of London, [ $1^{\mathrm{re}}$ éd. 1590], éd. J. S. Farmer, Londres, Tudor Facsimile Texts, 1911.

15 Thomas Lodge et Robert Greene, A Looking-glass for London and England, New-York, AMS Press, 1985

16 Cela n'empêche pas des auteurs ultérieurs comme Jonson ou Dekker de reprendre des abstractions plus ou moins réalistes, voir Stonex, op. cit., p. 195 et John Gross, Shylock, a Legend and Its Legacy, New-York, Londres, Simon \& Schuster, 1992, p. 49-50 et p. 63-67 : 
davantage sur les inventions dramaturgiques de Marlowe et de Shakespeare que sur leurs apports à la construction de la figure ellemême. De ce point de vue, Stonex affirme : "Marlowe's most fruitful contribution, [...] was the introduction of a rebellious daughter, a heroine who later was to become almost a dea ex machina both in the overthrow of her usurious father, the villain, and the salvation of her prodigal lover, the hero ${ }^{17}$ " . Mais il ne dit rien des scènes où Barabas ou Shylock apparaît, ni des jeux que cette apparition occasionne - jeux proches, à mon sens, de certains schémas de la comédie antique.

La cinquième scène du deuxième acte de The Merchant of Venice, par exemple, concentre de manière frappante une série de topoi de l'avarice au théâtre : enfermement de la fille, jalousie, plaintes à propos de la paresse du serviteur, rêve de sacs d'argent comme un présage de dommages ${ }^{18}$. La figure de l'usurier, être de raison, au jeu maîtrisé, à la mine sombre, est en quelque sorte parasitée par les « tics » de l'avare. Au cours de son procès, son attachement excessif à l'exécution de la lettre du billet prouve cette abdication de la raison au profit d'une passion vengeresse. Après la fuite de sa fille avec un chrétien, et la perte irrémédiable du fruit de son négoce, Shylock se laisse emporter par la passion « si désorientée 19 » (« so confused ») de la vengeance ; son langage se délite sous l'effet d'une parataxe émotive, lui qui pesait chaque parole, coordonnait strictement chaque pensée. Son discours devient haché, exclamatif, itératif et buté, réduit à trois thèmes : «ma fille !», « mes ducats ! », « mon billet ${ }^{20}$ ! », et par-dessus tout la « justice » :

Why there, there, there, there-a diamond gone, cost me two thousand ducats in Frankfort-the curse never fell upon our nation till now, I never felt it till now-two thousand ducats in that, and other precious, precious jewels... I would my daughter were dead at my foot, and the jewels in her ear! Would she were hearsed at my foot, and the ducats in her coffin! No news of them? Why, so-and I know not what's spent in the search: why, thou loss upon loss! The thief gone with so much and

Lucre, Mammon, Grip, Hornet, Bloodhound, Moth Interest, Perfidious Oldcraft, Sir Tyrent Thrift, sont quelques figures d'usuriers familières sur la scène élisabéthaine et jacobéenne.

17 Stonex, op. cit., p. 195-96.

${ }^{18} M V$, II.v.16-18 et sq.

${ }^{19} \mathrm{MV}$, II.viii.12 (Solanio).

${ }^{20}$ Il s'agit bien sûr du billet d'Antonio (« bond »). $M V$, III.i.39-42, voir l'ensemble de la scène ; III.iii.4-17. 
so much to find the thief, and no satisfaction, no revenge, nor no ill luck stirring but what lights o' my shoulders, no sighs but o' my breathing, no tears but o' my shedding.

(The Merchant of Venice, III.i.71-81)

Le chiffrement progressif de sa perte en or et en bijoux, puis des dépenses que sa fille a faites en chemin et des frais engagés pour sa recherche, les exclamations et interrogations sur son sort, le désespoir d'être maudit, l'accumulation des malheurs et leur surenchère, ne sont pas sans évoquer certains traits du monologue de Barabas dans The Jew of Malta ${ }^{21}$. D'ailleurs, Shylock fut interprété dans le même registre de jeu que le bouffon jusqu'à la fin du XviII ${ }^{\mathrm{e}}$ siècle ${ }^{22}$.

Dans l'Angleterre élisabéthaine, la possession des richesses et le choix du métier dans l'ordre communautaire et social étaient réglés, entre autres, par le code moral calviniste, que le puritanisme développa en vue d'une gestion utilitariste du temps et de l'argent, afin de s'opposer catégoriquement au désir des richesses, à la cupidité et à l'avarice ${ }^{23}$. Partant de la figure de l'usurier juif, toléré plus que réellement intégré à la société vénitienne (et anglaise ${ }^{24}$ ), Shylock tend à devenir une incarnation de l'avaritia, qui bascule de la raison à la folie, conséquence prévisible d'après la morale calviniste, puisqu'il agit uniquement en vue de son propre profit, et non pour la gloire de Dieu. L'agitation qui le perturbe le rétablit dans sa marginalité, dont la relative permissivité de Calvin sur les questions d'enrichissement aurait pu l'extraire. En effet, Calvin ne voyait pas d'inconvénient à ce que les prêtres s'enrichissent par le moyen du prêt à intérêt, car ils obtenaient ainsi un prestige souhaitable, s'ils restaient à l'abri des scandales 25 .

\footnotetext{
${ }^{21}$ Marlowe, The Jew of Malta, II.i.47-54. Mais Barabas retrouve de l'or et des bijoux cachés dans sa maison, c'est-à-dire une partie de la fortune qui lui a été confisquée, grâce à la complicité de sa fille Abigail.

${ }^{22}$ Voir John Wilders, éd., Shakespeare. The Merchant of Venice. A Casebook, Londres, MacMillan, 1969, p. 13.

23 Max Weber, L'Éthique protestante et l'esprit du capitalisme [Die Protestantische Ethik und der Geist des Kapitalismus, 1920], trad. de l'allemand par I. Kalinowski, " Champs classiques », Paris, Flammarion ( 3 ème édition), 2002, p. 253-54 et note 4.

24 Voir Weber, op. cit., p. 253, note 3.

25 Voir Weber, op. cit., p. 254, et Benjamin Nelson, The Idea of Usury, from Tribal Brotherhood to Universal Otherhood [1949], Londres, University of Chicago Press, 1969, p. XIX à XXV.
} 
Shylock, usurier juif ou avare christianisé, ou peut-on laïciser la Moralité ?

Shylock place son intérêt non dans la jouissance accumulatrice et absurde de l'or, mais dans le pouvoir inespéré (et presque désespéré) que sa détention confère, c'est-à-dire dans la capacité de contraindre son ennemi par la maîtrise des flux et des taux monétaires. Cependant, il serait faux de voir une quelconque stratégie dans la conclusion du contrat par Shylock; les aléas trop incertains auxquels il confie sa réussite - et dont il est conscient ${ }^{26}-$ le prouvent. Ce n'est que plus tard que la potentialité du billet éclatera, quand la fortune retourne la situation florissante d'Antonio. La force de l'intrigue se trouve précisément dans cette démonstration de la dynamique aléatoire de l'argent, qui peut attribuer et retirer le pouvoir sans être elle-même totalement maitrisable. L'usure de Shylock pose en fait un problème politique : par sa production exubérante et anarchique dans le circuit économique de «moneys », comme il prononce lui-même ${ }^{27}$, mettant au pluriel un terme normalement indénombrable, il concurrence l'autorité et les tentatives encore mal assurées de régulation des flux monétaires.

L'Angleterre élisabéthaine cherche en effet à se distinguer des autres empires expansionnistes, notamment de l'Espagne, célèbre (et fort décriée) pour ses fabuleux trésors, en concevant son rapport à la production de capital non en termes d'accumulation mais de circulation. La pratique du crédit s'intensifie et les formes de monnaie qui facilitent les transactions rapides (lettres de change, virements, etc.) se développent ${ }^{28}$. Tandis que le système mercantiliste européen s'appuie sur les réserves nationales d'or, l'Angleterre compte davantage sur le commerce extérieur pour la rentrée de devises, alors même que, de 1550 à 1680, l'inflation du cours de l'argent au gré des guerres ou des expéditions du royaume entraîne sa dévaluation et une thésaurisation croissante de l'or ${ }^{29}$. Parallèlement, le statut de l'usure

\footnotetext{
${ }^{26}$ Voir $M V$, I.iii.14-21. Il critique le « péril » que prend Antonio à dissiper sa fortune sur toutes les mers du monde.

${ }^{28}$ Fernand Braudel, Civilisation, économie et capitalisme ( $X V^{e}-X V I I I^{e}$ siècles), tome I : Les structures du quotidien : le possible et l'impossible, Paris, Armand Colin, 1979, p. 535.

${ }^{29}$ Braudel, op. cit., p. 524-527.
} 
évolue radicalement sous l'influence des idées calvinistes. D'après Benjamin Nelson, les moralistes chrétiens, gênés par le commandement du Deutéronome contre la pratique de l'usure entre « frères », c'est-à-dire membres de la même communauté, ont permis l'avènement d'une « altérité universelle » (« universal otherhood ») qui triomphera dans le capitalisme moderne où « tous sont 'frères' en étant des 'autres' à égalité $30 »$. L'usure pouvait donc théoriquement être pratiquée par tout le monde sans préjudice moral, et ne plus être systématiquement confiée aux Juifs. L'abrogation en 1571 du Bill Against Usury promulgué par Édouard VI permit finalement de pratiquer un taux d'intérêt à $10 \%$. The Essays or counsels, civil and moral de Francis Bacon (1597-1625) comprennent un court traité sur l'usure (XL) qui affirme la nécessité de sa pratique ${ }^{31}$; des documents prouvent que Shakespeare lui-même prêtait à intérêt à ses amis, alors que son succès au théâtre lui avait apporté la prospérité ${ }^{32}$.

L'usurier juif de Shakespeare se trouve à la croisée des remises en question de ces modèles économiques anciens. Thésaurisation et circulation sont les deux pôles de son comportement envers ses biens immatériels et matériels. Alors qu'il conserve jalousement tout ce qui le rattache à son passé, objets de mémoire ethnique ou familiale (bague de sa défunte épouse Léa, Jessica - héritière de sa lignée -, souvenirs du mépris d'Antonio envers son peuple), il est relativement détaché de la possession d'argent. En effet, Shylock apparaît moins comme un usurier intraitable que comme un vengeur des brimades dont les chrétiens accablent sa communauté. L'appréhension shakespearienne de sa judéité, qui s'apparente certes à une tradition satirique bien ancrée et régulièrement relancée par l'actualité ${ }^{33}$, lui offre une place centrale inédite, en prise avec les questionnements économiques, religieux, politiques, culturels de la fin du règne des Tudors. Il semble

\footnotetext{
30 Benjamin Nelson, The Idea of Usury, from Tribal Brotherhood to Universal Otherhood [1949], Chicago \& Londres, University of Chicago Press, $2^{\text {nd }}$ edition enlarged, 1969, p. XxIvXXV : "In modern capitalism, all are 'brothers' in being equally 'others' " [ma traduction].

$3^{1}$ Francis Bacon, Of Usury (1625), in The Essays or counsels, civil and moral (éd. Brian Vickers), Oxford, Oxford University Press, 1999, p. 93-96.

${ }^{2}$ Stephen Jay Greenblatt, Comment William est devenu Shakespeare [Will in the World : How Shakespeare became Shakespeare, 2004], trad. de l'anglais par Guy Rivest, Montréal, Édition Les Intouchables, 2007, p. 306-307.

33 Voir l'affaire Roderigo Lopez, médecin juif portugais attaché aux soins de la Reine, qui fut accusé d'avoir tenté de l'empoisonner, puis pendu en 1594. Voir NS, 1997, p. 1081 et Carsten L. Wilke, Histoire des Juifs portugais, Paris, Chandeigne, 2007, p. 138-139.
} 
chercher, tout au long de la pièce, à défendre un espace de survie à la fois géographique, juridique et économique dans la ville de Venise, confisqué par l'ascension des marchands chrétiens. Il tient davantage à la reconnaissance de ses droits, des souffrances de son peuple, voire à la revendication d'une certaine mémoire, qu'à la conclusion de marchés profitables et à la jouissance d'une prospérité chèrement acquise. Il se montre même méfiant contre son propre intérêt, et prend le risque de perdre un contrat juteux avec Antonio, qui jouit ouvertement d'une rare solvabilité. Il accepte de signer un billet peu valable pour son usure, «ni prisée, ni profitable» («not estimable, profitable neither $34 »)$. Jamais on ne le voit manipuler son or, ni même le compter ou l'inventorier pour l'exercice de son négoce; les trois mille ducats qu'il prête à Antonio sont eux-mêmes empruntés à son compatriote Tubal, il ne les possède pas chez lui 35 . On peut dire que cette discrétion est un effet somme toute logique de son avarice, une simple prudence impliquée par son métier ; mais il reste qu'il s'affirme clairement en tant que sujet, et non en objet d'une passion pour l'argent et l'or. Il est également significativement économe de sa présence : il n'apparait que dans cinq scènes sur vingt. Sa position sociale marginale et conflictuelle avec les marchands l'oblige sans cesse à calculer ses prérogatives, à lister ses biens, à garder « a tight grip on reality ", une forte emprise sur la réalité ${ }^{36}$.

Shylock ne gâche ni ses mots ni ses ducats. Mais quelques idiosyncrasies distinguent ses paroles, et révèlent des dispositions économiques troublantes: il emploie «moneys» au pluriel, mais encore « estimable » pour " valuable » à propos de la livre de chair ${ }^{37}$, mêlant les acceptions morale et économique des deux termes. Marc Shell assimile son vocabulaire à une « usure verbale » («a verbal usury »), c'est-à-dire à une perversion du langage sous forme de jeux de mots ou de tournures de flatterie ${ }^{3}$. Shylock propose de passer

\footnotetext{
${ }^{34} M V$, I.iii.162,

35 Ibid., 48-53.

36 “a tight grip on reality" (John Gross, op. cit., p. 66).

$37 M V$, I.iii.162, NS, p. 1100.

${ }^{38}$ Marc Shell, Money, Language and Thought. Literary and Philosophical Economies from the Medieval to the Modern Era, Berkeley, University of California Press, 1982, p. 49 On retrouve ce terme dans le Talmud, chez les Pères de l'Église et dans les Traditions de l'Islam.
} 
contrat en jouant sur les valeurs chrétiennes de l'amitié : il offre « cette » amitié dont il est témoin, celle d'Antonio et de Bassanio, pour en faire profit, et « achète » la faveur d'Antonio qui le méprise : " to buy his favour, I extend this friendship 39 ». Cette « usure verbale » s'oppose au sens de la valeur comprise comme une compétence pour la mesure et le discernement des hiérarchies morales, l'amour et l'amitié prévalant sur l'intérêt économique, le profit personnel et immédiat.

Les scènes du choix des prétendants entre les trois coffrets d'or, d'argent et de plomb pour obtenir la main de Portia, la riche et belle héritière de Belmont, révèlent la désuétude d'un idéal aristocratique de monstration de sa propre valeur (par le don ostentatoire de soi ou de son or), mais aussi les difficultés de l'argent, objet encore mal maîtrisé, qui attise traditionnellement les méfiances, à combler cette disparition de l'ancien critère.

Les métaux, tous trois gravés d'une inscription, comme le sont monnaies et alliances ${ }^{40}$, sont les moyens choisis par le père de Portia pour contracter le mariage. Il a déplacé le choix de l'amour, trop soumis aux intempérances de la passion pour être fiable, sur celui d'un métal, dont la neutralité n'est qu'apparente. Un authentique vir comme ce père, un homme qui allie vertu morale et respect des préceptes religieux, ne peut se tromper sur la justesse de son entreprise malgré son apparence aléatoire, et de même le marchand mû par des valeurs supérieures, même si son commerce est sujet à la dérive des monnaies, ne peut qu'obtenir de bons profits. Élaboration d'un esprit supérieur guidé par Dieu, l'énigme posée par le père permettra un jugement «juste » (« rightly», I.ii.28), conformément à son intention, la droiture du prétendant et l'inclination de la fille. Mais le moyen par lequel le mariage va se décider demeure ambigu: chest signifie « coffre» et «poitrine», indiquant que ces hommes qui se présenteront devant les coffrets seront tenus de juger selon le cœur de Portia : le contenu (le cœur de la belle) sera remporté par qui saura reconnaître le contenant digne d'elle, quelle forme représente sa valeur. Portia sait le peu de vigueur des règles formulées par la raison :

$39 M V$, I.iii.164, NS, p. 1100 (c'est moi qui souligne). La plupart des commentateurs comprennent "this friendship » comme l'amitié que Shylock offre à son ennemi ; il se pourrait cependant que Shylock joue encore sur les mots et « offre » l'amitié d'Antonio et de Bassanio à sa quête de profit.

${ }^{40}$ Ce rapprochement est indiqué par Marc Shell, op. cit., p. 56. 
« The brain may devise laws for the blood, but a hot temper leaps o'er a cold decree. Such a hare is madness, the youth, to skip o'er the meshes of good counsel, the cripple 41 ». Le père connait aussi ces «tempéraments» prompts à contracter mariage sous le feu de la passion pour obtenir la gloire, l'argent ou les femmes. Puisqu'on ne peut juger un homme que selon son cœur, au moins ses débordements seront-ils habilement maîtrisés, grâce à la sagesse paternelle, par un stratagème qui prouvera, en même temps qu'un réel amour, l'adhésion du prétendant aux valeurs de Belmont, et surtout sa capacité à les reconnaître, donc à les attribuer à son tour justement. Ainsi c'est une véritable «compétence» (competency), au sens étymologique de « proportion, juste rapport », d'où dérive le sens moderne de « capacité fondée de jugement », qu'exigent Portia et son père du futur amant. La « compétence », c'est-à-dire la mesure, est en effet garante d'une « vie plus longue » : " It is no mean happiness [...] to be seated in the mean. Superfluity comes sooner by white hairs, but competency lives longer 42 ». C'est ici Nerissa, la fidèle confidente, qui en fait l'éloge. Mais «mean» signifie aussi bien «pauvre, vil» et «avare», que « moyen terme, milieu », mais aussi « commun, universel 43 », selon le contexte et l'intention de l'interlocuteur. Le mot pointe une ambiguïté dans le langage même, où s'engouffrent toutes les incertitudes d'une époque à propos de ce « juste milieu » et de la « compétence » pour la mesurer.

L'enjeu de l'énigme pour les prétendants est donc le suivant : prouver leur «compétence » à Portia, c'est-à-dire mesurer proportionnellement le rapport entre le métal, l'inscription et le contenu de chaque coffret. Le point de vue que chacun adoptera sera déterminant dans leur raisonnement et leur choix final, puisqu'il montrera dans quel système de valeurs il s'inscrit. Un «pauvre plomb » est finalement préféré aux métaux précieux comme symbole d'amour véritable. L'or, valeur toujours garantie dans le domaine du fantasme amoureux - ainsi les « boucles brillantes » (« sunny locks »,

\footnotetext{
${ }^{41} M V$, I.ii.15-18.

${ }^{42}$ Ibid., v.6-8.

43 Voir l'article «Mean, adj. 1 et 2 » dans Oxford English Dictionary, Oxford, Oxford University Press, 2001 [en ligne : http://www.oed.com/view/Entry/115438?. Consulté le 8 février 2013].
} 
I.i.169) de Portia sont-elles comparées à la Toison d'or 44 -, est concurrencé par des formes plus souples (petites pièces, métal pauvre, mais aussi lettres et billets) dans une nouvelle dynamique amoureuse et économique, fondée non plus sur le faste et l'apparat du trésor, mais sur la fluidité de l'échange. Quant à l'argent, choisi significativement par le prince espagnol, il n'assume plus l'objectivité du pur moyen d'échange, ni la stabilité d'un métal précieux et thésaurisable. L'inscription sur laquelle le choix du prince se fonde pour sa décision (« Who chooseth me shall get as much as he deserves », II.ix.36) ne garantit plus l'obtention du « mérite », pas plus que la frappe du métal par une autorité ne garantit la validité de son cours, toujours soumis aux dévaluations des États pour le « bien » politique.

La perversion du langage économique par Shylock en vue de son plus strict intérêt, et l'éclat déraisonnable de son avarice révèlent l'absurdité d'une mainmise autoritaire sur la vie économique, mais suggèrent aussi les tensions qu'engendre le maintien d'un idéal aristocratique et chrétien dans l'évolution libre des marchés. Shakespeare construit une figure moderne en remotivant des traits appartenant au comique de l'avarice, être de thésaurisation, sclérosé dans une mémoire familiale et ethnique impossible à satisfaire, dans la question cruciale de l'usure et de son statut en passe d'être accepté dans la société. Ce faisant, il laïcise en quelque sorte la Moralité, car le débat, une fois la folie de l'usure rejetée du côté du délire avare et transformée en fièvre de possession d'un bien encore plus immatériel et impalpable que l'argent, se concentre durant toute l'intrigue sur la recherche d'un critère économique de la valeur qui soit à peu près en accord avec les principes chrétiens de don non profitable et d'amitié, mais aussi avec la dynamique de l'échange marchand, devenu incontournable dans toute relation sociale. Cette question travaille aussi le texte lui-même, comme nous allons le voir à présent. The Merchant of Venice propose, à notre sens, une tentative de refondation du genre comique, déstabilisé par la tendance tragique de la comédie shakespearienne: les propos de Shylock et des marchands vénitiens sont en effet présentés avec autant de force et de conviction, du moins avant les malheurs de Shylock et son basculement du côté de la " passion » de l'avarice, trait propre à la tension tragique.

${ }^{44} M V$, I.i.167-172. 


\section{Redéfinition d'un genre comique et interprétation d'un texte}

The Merchant of Venice tient, d'après Walter Cohen, une place atypique dans la comédie contemporaine, à cause de la "gravité » de son sujet et de son «emphase socio-économique 45 ». Elle montrerait surtout «les limites formelles et idéologiques » d'un genre ${ }^{46}$. Il est possible de poursuivre cette réflexion en observant notamment le dénouement de la pièce, à savoir la scène du procès et quelques aspects de l'acte $v$.

L'intervention de Portia en docteur de la loi, figure censée interpréter correctement ses préceptes, annonce une conception particulière de la forme de cette interprétation ${ }^{47}$. Shylock réclame la justice sur un mode tragique : «I stand for judgement » (IV.i.102) ; «I stand here for law» (IV.i.141), mais elle se présente sous une forme déguisée, cachée, bref comique. Une lettre avertit de l'arrivée d'un jeune docteur envoyé par un célèbre avocat (IV.i.162) ; c'est ce même artifice de la missive inattendue qui annoncera la perte des vaisseaux d'Antonio, puis la nouvelle de leur sauvegarde miraculeuse. Les circonstances des apparitions de ces lettres relèvent toujours de la surprise, comme un ultime recours alors que tout semblait désespéré. La lettre, moyen de la circulation des nouvelles et du sens entre les hommes, se comprend alors dans une structure ternaire dont le pendant positif, dans la configuration marchande, est la monnaie comme moyen de circulation des valeurs ${ }^{48}$, et le pendant négatif le billet comme arrêt et fixation du sens. Portia, en s'appropriant la voix

45 Walter Cohen, «The Merchant of Venice and the Possibilities of Historical Criticism », English Literary History, 49, 1982, p. 765-789, et repris dans Martin Cole, éd., The Merchant of Venice. Contemporary Critical Essays, coll. "New Casebooks", New-York, St Martin's Press, 1998, p. 61. "The work stands apart from Shakespeare's other comedies of the 1590 s, romantic or not, and, in addition, from most other comedies of the period, both in the gravity of its subject and in its socio-economic emphasis".

${ }^{46}$ Cohen, op. cit., p. 62. "The Merchant of Venice, by its very atypicality, reveals the formal and ideological limits of Renaissance romantic comedy”.

47 De nombreuses études et commentaires de légistes ont paru à propos de ce procès, ainsi que du contrat. Ils relèvent tous la position partiale de Portia dans cette affaire, puisqu'elle est fiancée à Bassanio, l'ami du débiteur de Shylock, et protectrice de Jessica, la fille de l'usurier qui s'est réfugiée à Belmont. Pour une revue complète de cette critique depuis le XIX ${ }^{\mathrm{e}}$ siècle jusqu'aux années 1960 environ, voir Owen Hood Phillips, Shakespeare and the Lawyers, Londres, Methuen, 1972, p. 91-118.

48 Voir Jean-Joseph Goux, Frivolité de la valeur. Essai sur l’imaginaire du capitalisme, Paris, Blusson, 2000 ; Freud, Marx. Économie et symbolique, Paris, Seuil, 1973 ; Marc Shell, op. cit., 1982. 
de la justice, détourne la loi, symbolisée par le livre, forme fixe de l'écrit, au profit d'Antonio, par une lettre. De même, Shylock a détourné le profit économique selon son intérêt par un billet écrit comme dans le « métal », matière à laquelle Gratiano compare la haine du juif (IV.i.123-125).

Dans cette lettre qui l'annonce, il est expliqué à la Cour que le docteur a « feuilleté bien des livres 49 » avec Maître Bellario pour tenter de résoudre par la certitude de la science légale l'affaire qui oppose Antonio à Shylock ; c'est aussi au livre de la loi que Balthazar / Portia a recours pour contrer la première sentence favorable à Shylock (Iv.i.343-357). La performance de sa parole repose sur ces deux supports, le livre et la lettre, alors que l'un ou l'autre fait toujours défaut aux autres protagonistes du procès.

Cette ambivalence du support de la parole lui permet de changer de stratégie au cours de l'audience. Entrée par l'entremise d'une lettre, Portia a d'abord tenté de rétablir une négociation entre les deux hommes, en incitant Shylock à prendre les six mille ducats que lui propose Bassanio, et à se montrer clément envers Antonio (Iv.i.177229). Puis la loi vénitienne et ses décrets ${ }^{50}$, dont elle aurait consulté les nombreux textes, lui servent à affirmer la puissance de la parole judiciaire, récupérant dans le sens de la justice la tentative de Shylock de détournement de la parole d'Antonio à son profit, par le recours à la même rigueur, c'est-à-dire à l'interprétation de la loi au mot près :

\footnotetext{
This bond doth give thee here no jot of blood.

The words expressly are 'a pound of flesh'.

Take then thy bond. Take thou thy pound of flesh.

But in the cutting it, if thou dost shed

One drop of Christian blood, thy lands and goods

Are by the laws of Venice confiscate

Unto the state of Venice.
}

(IV.i.301-307)

Il y a donc substitution, dans la scène du procès en particulier, des formes de validation de l'écrit aux formes monétaires problématiques, avec les mêmes rapports d'opposition entre formes "dures » et formes "souples » : ducat (métal) / billet (papier), livre /

\footnotetext{
49 "We turned o'er many books together" (IV.i.154).

${ }^{50} \mathrm{MV}$, IV.i.173 et 213-214.
} 
lettre. Cette transposition des problèmes liés à la sphère économique sur le plan de la forme serait-elle une tentative de revalorisation de l'économique à travers le texte théâtral et sa mise en scène ? Ou bien tendrait-elle à rendre ce dernier caduc, comme un effet pervers de l'innervation de l'économie dans la forme littéraire (et le genre théâtral)?

En effet, ce rapprochement final de l'usage de la lettre pour un profit certes altruiste et généreux, mais associé à la ruse et à la merveille par son apparition artificielle, non déterminée par la logique de l'intrigue, et de l'usage intéressé de l'argent pour un profit personnel réprouvé puis condamné, soulève des questions sur l'action de la parole qui soutient le premier usage. Ce dénouement comique enchevêtré semble avertir le lecteur qu'il existe là une difficulté, que la décision judiciaire semble résoudre, mais qui n'est peut-être en réalité solvable que par un choix de mise en scène. Au cours de la démonstration rigoureuse de la culpabilité de Shylock selon les décrets de Venise, Portia use de tours rhétoriques qui insistent non seulement sur la performativité de la parole du docteur de la loi ${ }^{51}$, mais encore sur sa capacité à faire jaillir la justice de la bouche même de qui veut tirer de la loi un profit financier. Portia se réfère à la stricte lettre du billet, comme le fait Shylock. La validité de sa parole est donc soumise au même doute.

Comment dès lors rendre une valeur morale à la parole, et par conséquent fixer la place de l'argent dans l'ordre social ? En en revenant paradoxalement à la matière de la monnaie, et à celle du livre. La compilation, de ducats sonnants et trébuchants, et des feuillets du livre qui constituent les décrets de Venise ${ }^{2}$, par des personnages dont la vertu chrétienne ne fait pas de doute pour les spectateurs, redonne toute sa certitude à leur valeur. La qualité des pièces qu'on dépense, des livres qu'on lit, dépend de celle des personnes qui les manipulent, et surtout de l'usage que celles-ci en font, de leur interprétation,

${ }^{1}$ Ibid., 197-198 : "I have spoke thus much / To mitigate the justice of thy plea", dit-elle à Shylock; Ibid., 1l. 352 : "In which predicament I say thou stand'st...”, énonce-t-elle avant de décliner les griefs que la justice retiendra contre lui. Le duc, autre figure d'autorité judiciaire, seconde le jugement de Portia en ajoutant sa parole à la sienne : "He shall do this, or else I do recant / The pardon that I late pronounced here" (ibid., 386-387).

$52 \mathrm{La}$ " coutume » anglaise n'est pas, quant à elle, un droit écrit. Voir René David et alii, Introduction à l'étude du droit privé de l'Angleterre, Paris, Librairie du recueil Sirey, 1948. 
généreuse et désintéressée, motivée par une amitié et des codes moraux communs. Mais cette interprétation s'étend, en dernier recours, à autrui, via la parole de clémence du duc, qui avait soutenu la décision de Portia, et d'Antonio. Le duc, pour signifier « la différence de [son] esprit » («the difference of our spirit »), épargne la vie de l'usurier, et redistribue ses biens à sa famille (Lorenzo et Jessica) et à l'État, ce à quoi Antonio ajoute la demande de conversion (Iv.i.363385). Économie, justice, morale et religion sont donc réconciliées sous le joug de l'autorité - sous forme de livre à interpréter -, de l'amitié, et de la piété des citoyens de Venise.

Le redressement des valeurs se poursuit lors des scènes suivantes, du point de vue cette fois-ci de l'échange amoureux. Bassanio a été capable de donner six mille ducats par amitié, mais il conçoit le jugement du docteur comme un service méritant récompense. Les marchands se disent «endettés » («indebted », IV.i.409, 417-19) et greffent donc encore une valeur marchande et intéressée supérieure à la valeur désintéressée que confère l'amour. Après quelques hésitations, Bassanio donne la bague que sa fiancée lui a remise, comme « tribut, non comme salaire 53 » (« as a tribute, / Not as a fee ») à l'avocat. Ce dernier lui avait pourtant assuré que la libération d'Antonio lui suffisait comme salaire à la hauteur de son mérite, en employant ironiquement un langage fruste et « mercenaire » :

He is well paid that is well satisfied,

And I, delivering you, am satisfied,

And therein do account myself well paid

My mind was never yet more mercenary 54 .

Ainsi, le jugement sur la hiérarchie de la valeur reste une question des plus difficiles à trancher, puisqu'elle dépend, comme le verdict de Shylock (IV.i) ou la beauté d'une musique au clair de lune (v.i), des « circonstances » (« respect », v.i.98) d'où ils émergent. C'est pourquoi la position et le discours de Bassanio pour expliquer son geste à Portia le « divisent » (« double self », v.i.242-43). Mais la juste rétribution du mérite vaut apparemment moins que la fidélité à la

$53 M V$, IV.i.409, 417-19.

54 Ibid., 411-414 (c'est moi qui souligne). 
personne aimée. La série de surenchères (« outface », « outswear », IV.ii.17, « outnight », v.i.23) à laquelle se livrent Portia, Nerissa et même Jessica forme une dernière joute rhétorique pour faire triompher la valeur chrétienne de l'amour et des personnes, contre celle, économique, du marché et des objets, grâce à un langage qui convient, chez Shakespeare, aussi bien à l'un qu'à l'autre 55. Le langage économique sert bien, en dernière instance, à justifier la prévalence d'une valeur absolue et non négociable ; il est à son tour détourné par Portia, pour le seul profit personnel qui vaille, à savoir l'intérêt du cœur. Il justifie également, entre les lignes, le bien-fondé de la comédie, qui s'achève par la réconciliation traditionnelle des couples.

Les alliances finales, nuptiales et économiques, sont scellées par l'échange renouvelé d'anneaux et de lettres. Les matières, métallique et littéraire, sont ainsi réinvesties par de nouvelles valeurs positives ${ }^{56}$. L'anneau du mariage n'est ni un objet de luxe qui porte en lui une valeur marchande exorbitante et dont la possession constitue une richesse en soi, ni un simple instrument économique, interchangeable, et dont le prix est imposé ; il est le symbole de l'union amoureuse, d'un échange de parole et de fidélité ${ }^{57}$. Portia remet la bague à Bassanio, la lettre qui le rétablit dans ses biens à Antonio, comme une dispensatrice de «manne» féconde ${ }^{5}$. Ainsi associée au don mutuel des alliances, qui remotive la qualité du métal, la lettre, et son contenu heureux, n'est plus une fixation définitive du sens, puisqu'une lettre peut en invalider une précédente. Certes elle reste un subterfuge, et un artifice. Mais il semble que Shakespeare choisisse ici de magnifier cette artificialité, dans le cadre d'un théâtre capable de construire une esthétique nouvelle de la valeur, en insistant sur la circulation de la lettre comme

\footnotetext{
55 Sur cette spécificité du langage shakespearien voir Shell, op. cit., passim.

${ }^{56}$ Sur l'identité de la lettre et de la monnaie, voir entre autres Schacht, Joachim, Anthropologie culturelle de l'argent: le masque mortuaire de Dieu [Die Totenmaske Gottes. Zur Kulturanthropologie des Geldes, 1967], trad. de l'allemand par Joseph Feisthauer, Paris, Payot, 1973, p. 83; Goux, op. cit.; Mark Netzloff, "The Lead Casket: Capital, Mercantilism, and The Merchant of Venice”, in Linda Woodbridge, éd., Money and the Age of Shakespeare: Essays in New Economic Criticism, New-York, Palgrave Macmillan, 2003, p. 159-176.

57 Notons cependant, avec Joachim Schacht, que la monnaie a pu prendre primitivement des formes annulaires ou discales, symboles de perfection, d'éternité et de "solidarité sociale », valeurs inhérentes à la sacralité du monnayage et de la circulation de l'argent (op. cit., p. 77).

$5^{8} M V$, v.i.265-278.
} 
métaphore dramatique d'un véhicule privilégié du sens et non comme support linguistique neutre mais dont le sens varie en fonction, encore une fois, de l'angle sous lequel on le lit. Sauvetage là encore in extremis d'un genre comique qui a pourtant basculé dans le tragique du point de vue de la valeur, grâce à un artifice théâtral réinterprété dans un sens positif.

Shylock fonctionne donc comme une conscience économique et dramatique harcelante, non sous la forme évanescente du fantôme, mais en incarnation complexe et irritante d'une mémoire théâtrale, morale, religieuse et économique problématique en cette période de naissance de l' « esprit capitaliste », qui peine à définir la valeur et la place à accorder à l'argent dans les relations humaines. C'est, voulonsnous croire, à travers son appréhension de l'argent que Shylock met en cause la légitimité des actions des chrétiens engagés sur la voie du capitalisme marchand. L'attachement de Shylock à la rigidité du métal, à la rigueur de la loi, ou enfin à la lettre du billet, retranscrit triplement une attitude « archaïque » et péremptoire, associée à l'interprétation de la Bible hébraïque et à une mémoire familiale et historique douloureuse. En fixant définitivement le sens du billet et de la lettre, les souples moyens modernes de la transaction financière, il interdit l'échange, n'acceptant que l'usure, activité réservée aux ressortissants de son peuple. Portia doit donc réaffirmer, grâce à une ruse, la relativité interprétative de la lettre (et de son contenu, l'écrit), comme symbole (chrétien) de cet échange clair, sûr et mesuré. Reste que cette nouvelle « loi », énoncée à partir de valeurs transcendées par l'amitié qui unit les royal merchants chrétiens, ne peut se définir qu'entre les lignes d'un texte, destiné à occuper une autre forme de mémoire, celle de l'interprète du rôle dans l'avenir. Il semble que Shakespeare, dans The Merchant of Venice du moins, explore les vertus cathartiques d'un théâtre pleinement littéral, c'est-à-dire dont le texte, grâce à l'interprétation qu'en font comédiens et lecteurs (ou spectateurs), redonne un sens à la comédie qui a dévoilé sans équivoque le bouleversement contemporain des valeurs, contribuant à en faire une œuvre capable de marquer la mémoire et l'imaginaire modernes sur l'argent, l'avarice et l'usure.

Leur figuration doit ainsi dorénavant compter avec la dimension médiatique de l'argent, concurrence que le théâtre comique 
shakespearien entend dépasser en «rationalisant» ses propres moyens. The Merchant of Venice propose donc un réel réinvestissement de l'échange économique pour une éventuelle acclimatation de son principe moderne. D'après Christian Biet, le théâtre offre en effet un intérêt personnel et axiologique qui se transmet au gré d'une dépense d'énergie commune ${ }^{59}$. Grâce à lui, l'échange économique trouve ici une autre valeur. Mais si Shylock exploite la souplesse moderne de l'argent, il reste soumis aux fluctuations de sa valeur; Shakespeare semble vouloir affirmer par contraste la vertu stabilisatrice de l'art théâtral.

\section{Tatiana BURTIN}

Université de Montréal / Université de Paris Ouest Nanterre La Défense

59 Voir Christian Biet, "'Argent contre chair', 'Argent contre plaisir'. Le théâtre comme actualisation, figuration et dépassement de l'échange économique », in Martial Poirson, Yves Citton et Christian Biet, éds., Les Frontières littéraires de l'économie (XVII ${ }^{e}-X I X^{e}$ siècles), coll. " Littérature \& idée », Paris, Desjonquères, 2008, p. 201-214. 